\title{
Understanding and Addressing Aggressive and Related Challenging Behaviors in Individuals with Dementia
}

\author{
By Dimitra Loukissa*
}

\begin{abstract}
Dementia in the older adult population is a multifactorial, complex condition currently affecting an estimated 35.6 million individuals worldwide. The literature has been consistent highlighting the financial, emotional, and social burden of the illness on the affected individual, the family, and health care resources. Although in more recent decades there have been significant advances in the area of genetics, neuroscience, testing, and psychopharmacology, the management of this complex condition continues to present major challenges. Common illness related challenging behaviors may range from wandering, restlessness, irritability, personality changes, hoarding, sexual inhibition to escalating anxiety, verbal and physical agitation and aggression. Current pharmacological and non-pharmacological interventions aim at addressing behavioral and psychological symptoms to alleviate patient anxiety, promote cognitive stimulation, address safety issues, decrease caregiver burden, and empower healthcare personnel to better care for these patients in the hospital and nursing home settings. This article reviews published literature covering a 10-year period up to spring 2017 utilizing the PubMed, CINAHL, MEDLINE, Cochrane and ProQuest databases. From the initial 242 identified articles, twenty one studies examining nonpharmacological interventions were deemed appropriate to be reviewed. Key words included dementia, disruptive behaviors, and nursing interventions. Criteria for studies reviewed included: peer-reviewed articles published in the last 10 years, written in English, with the focus on intervention implementation. Findings from those studies offer insights into a variety of approaches that can address challenging behaviors in dementia. Advancements in the areas of behavioral, psychological, milieu interventions and also most commonly utilized pharmacological agents are discussed, as well as the importance of nursing staff involvement in identifying escalating behaviors and successfully intervening to promote safety, and decrease anxiety and agitation. Finally, implications for practice are explored.
\end{abstract}

Keywords: Dementia, Disruptive behaviors, Nursing interventions, Older adults.

\section{Introduction}

Dementia, with Alzheimer's disease being the most prevalent form of the illness, is the $6^{\text {th }}$ leading cause of death in the USA and affects an estimated 5.2 million Americans, the vast majority of them over the age of 65 . It is estimated that by 2050 this number will escalate to 13.8 million cases (Fargo and Bleiler 2014). Global estimates report that currently there are 46.8 million individuals worldwide with a diagnosis of dementia. It is expected that cases will double by 2030 to approx. 74.7 million and by 2050 the affected individuals will account for 131.5 million worldwide. In the USA alone the cost of care such as medical services, caregiving services, hospitalizations, adult day services, long-term care, and hospice care services, is estimated at \$214 billion. Current worldwide estimates raise the cost to $\$ 818$ billion (Fargo and Bleiler 2014).

This illness which results in progressive decline in function and cognition, extends over a period of several years, and causes significant distress to the affected individual, their family, hospital personnel and the society at large. Over

*Professor, North Park University, USA. 
the course of the illness on average $90 \%-97 \%$ of the affected patients experience at least one aggressive symptom whether they live in the community or in a nursing home (Cankurtaran 2014, Ballard and Corbett 2010). Although there is no definitive treatment for dementia, great progress has been made over the past couple of decades in understanding the complexity of the brain and illness symptomatology as well as implementing non-pharmacological and pharmacological interventions to control the aggressive behaviors.

This work reviews current literature on individual and milieu behavioral interventions to manage disruptive behaviors including basic pharmacological approaches that can be considered as adjunct interventions to relieve persistent aggressive behaviors. Clinical implications and recommendations for nursing practice are also discussed.

\section{Disruptive Behavior Identification in Dementia}

Throughout the course of the illness behaviors represent changes of the disease process at the molecular, cellular, and neurochemical levels. According to the literature, behavioral disturbances have been classified as primary and secondary exhibiting various degrees of intensity, at times involving psychiatric symptomatology (Desai and Grossberg 2001, Kales et al. 2014a). These behaviors can occur at home, the community, nursing home settings, but also during hospitalization (Table 1).

Table 1. Common Disruptive Behaviors in Dementia

\begin{tabular}{|l|c|c|}
\hline Primary Behaviors & $\begin{array}{c}\text { Secondary Behaviors } \\
\text { Result of }\end{array}$ & $\begin{array}{c}\text { Psychiatric } \\
\text { Symptomatology }\end{array}$ \\
\hline $\begin{array}{l}\text { Cognitive Challenges } \\
\text { Forgetfulness, concentration problems, } \\
\text { sundowning, impulsivity, inability to care } \\
\text { for self, poor judgment }\end{array}$ & Medication side effects & Paranoia \\
Affective Challenges & Delusions \\
$\begin{array}{l}\text { Apathy, low motivation, lack of interest, } \\
\text { irritability, agitation (yelling, wandering, } \\
\text { hoarding, screaming, cursing, } \\
\text { disinhibition), aggression (grabbing, } \\
\text { biting, pushing) }\end{array}$ & $\begin{array}{c}\text { Misinterpretation of the } \\
\text { environment }\end{array}$ & Hallucinations \\
& Misidentification & Anxiety \\
& Insomnia & Depression \\
\hline
\end{tabular}

Primary Disruptive Behaviors include behaviors that range from escalating anxiety, sundowning, pacing, wandering, to refusal to redirection, verbal threatening, yelling, screaming, and hoarding. Other disruptive behaviors may include sexual inhibition, physical threats or harm to others by kicking, grabbing, biting, pushing through or physically attacking someone (Duane et al. 2015).

Secondary Disruptive Behaviors usually are the result of medical conditions, medication side effects, pain, misidentification and misinterpretation of the 
environment and at times delirium related to infection or other acute medical conditions.

Psychiatric Type Symptomatology may include paranoia, delusions, hallucinations, depression, anxiety, and psychosis.

Researchers have stressed the importance of the use of measurement tools to objectively determine the severity of the behavior which may result from depressive symptomatology, wandering, psychotic presentation, or aggression as such behaviors are the most challenging to address (Derlinde et al. 2014). A total of 83 measurements designed to measure challenging behaviors in dementia were examined by Van DerLinde et al. (2014). The researchers commented that only few of those instruments assessed reliability and validity. Although reliability and validity were satisfactory when reported, it is strongly suggested that when considering the use of an assessment tool, variables such as study sample, target population, living situation, time frame, and setting are included as they can affect the outcomes.

\section{Impact of Dementia Disruptive Behaviors}

Common challenging behaviors in dementia create safety issues for the affected individual and those around them. Symptomatology can vary in presentation and intensity over the course of the illness. Zwijsen et al. (2014) argue that over $80 \%$ of demented nursing home residents express at least one form of disruptive behaviors. Those behaviors may range from repetition, wandering, mood related outbursts, swearing, hoarding, refusing to attend to hygiene, eating problems, disrobing, inappropriate sexual behaviors, to paranoia, hallucinations, delusional thinking, irritability, anger, verbal and physical aggression. Backhouse et al. (2014) found aggression to be the most challenging behavior in nursing home residents, as reported by $37 \%$ of care home managers.

Apart from safety and quality of life issues for the affected individual and increase in caregiver stress, as well as threats to caregiver income and health, unaddressed disruptive behaviors usually accelerate the progression of the illness (Van Den Wijngaart et al. 2007, Rabins and Lyketsos 2011).

\section{Case Study}

Mr. Jones, a 75-year-old Caucasian retired male, with a recent diagnosis of dementia, was a successful administrator of a large company and avid reader. During his career, Mr. Jones had travelled all over the world, maintained a healthy life style and participated in volunteer work in his community. Several months ago his spouse became concerned as Mr. Jones was becoming increasingly resistant to redirection, and on a couple of occasions he became aggressive with her, pushing her away from the door so he could exit the house and "go to work". As Mr. Jones was becoming increasingly difficult to control the decision was made to have him hospitalized to have his impulsivity and aggressive behaviors addressed. During his hospitalization on a general psychiatric unit, Mr. Jones required a couple of 
times restraints as he was physically aggressive with staff for not allowing him to exit the unit. New medication prescriptions addressed the issue of aggression for the most part, however, Mrs. Jones was concerned that her husband "wasn't the person she knew anymore" as he appeared sedated and disinterested most of the time. Mr. Jones was transferred to an inpatient dementia unit for rehabilitation and further treatment and stabilization, where he was able to benefit from a patientcentered approach, a milieu that was designed to address the needs of individuals with similar presentation and a daily structure and routine.

\section{Interventions}

A typical regimen to address disruptive behaviors includes a combination of antipsychotic agents to control dangerous and aggressive behaviors in combination with non-pharmacological interventions and environmental modifications in the inpatient setting. Although there is a general agreement amongst health care providers that disruptive behaviors must be addressed successfully, behavioral interventions tend to be much more preferred over the use of antipsychotic agents which have been widely criticized.

In more recent years, regulatory requirements have achieved a $3 \%$ reduction of antipsychotic medication in nursing homes over a three year old period, as more focus is placed on the use of non-pharmacological interventions and personcentered care (Benbow 2014, Blackburn and Bradshaw 2014). The American Geriatric Society places emphasis on the worldwide initiative for emphasis on the use of non-pharmacological approaches as the first line of interventions to address challenging behaviors (Anand et al. 2014). According to the Health and Social Care Information Center in Great Britain there has been a $17 \%$ reduction in the use of antipsychotics in British nursing homes over the course of the past decade (Prince et al. 2013). Yet, due to the conservative response to non-pharmacological approaches, antipsychotic medications often may dictate the course of action in maintaining safety. Although preference is given to behavioral interventions, it is not uncommon that Child et al. (2012) found that $26 \%$ of nursing home residents were receiving antipsychotic medications.

Due to the lack of a single treatment modality that successfully addresses disruptive behaviors, the availability of non-pharmacological and pharmacological interventions, although with various degrees of effectiveness, has allowed caregivers to manage some of those behaviors in the community, thus, delaying institutionalization, and allowing the affected individual to stay in their familiar environment, which in return limits costs, maintains quality of life and prevents caregiver burden. Backhouse et al. (2014) found that concurrent use of nonpharmacological interventions along with the use of antipsychotic medications was effective in addressing disruptive behaviors in care homes. The case study presented above is a clinical testimonial that the use of both pharmacological and non-pharmacological interventions is far more superior in outcome effectiveness than just one approach. Other treatment modalities with various degrees of effectiveness may include Electroconvulsive Therapy or even the use of restraints 
in emergency situations as presented in Table 2, in cases where safety is significantly compromised.

\section{Non-Pharmacological Interventions}

Current practice guidelines place a great emphasis on the use of nonpharmacological approaches as an initial intervention before resorting to chemical agents as they offer viable and in a number of cases successful alternatives. Although intervention effectiveness in many cases might be modest, there has been ongoing discussion in the literature on interventions that support social interactions for patients affected by dementia, person-centered care approaches as well as the benefit of alternative interventions such as aromatherapy, physical activity, and pet therapy to name a few (Kramer et al. 2009, Nordgren and Engstrom 2014, Tournier et al. 2017, Forrester et al. 2014, Soares-Weiser 2014). Table 3 summarizes related studies.

The benefit of short, structured but systematically applied tactile activities tailored to specific needs of the individual using a "tool box" was discussed by Cohen-Mansfield et al. (2011a). Along the same lines preliminary evidence from small studies assessing aromatherapy shows that the use of lavender oil and Melissa officinalis oil (lemon oil) in particular were successful in decreasing agitation while offering the added benefit of a safe alternative (Ballard and Corbett 2010).

To address mild to moderate depressive symptomatology Ballard and Corbett (2010) discuss the benefit of simple activities such as individual or group exercise (Figures 1 and 2), singing, and reminiscing. The benefit of doll therapy as a means of providing comfort, identity, attachment, and sensory stimulation has been widely criticized. Yet, small studies have reported benefits in the areas of comfort, wellbeing, engagement, decrease in agitation, and in some instances increase in dietary intake, as well as creating a sense of active participation in the environment, rather than passively receiving care (Benbow 2014, Mitchell 2014).

Figure 1. Exercise Therapy

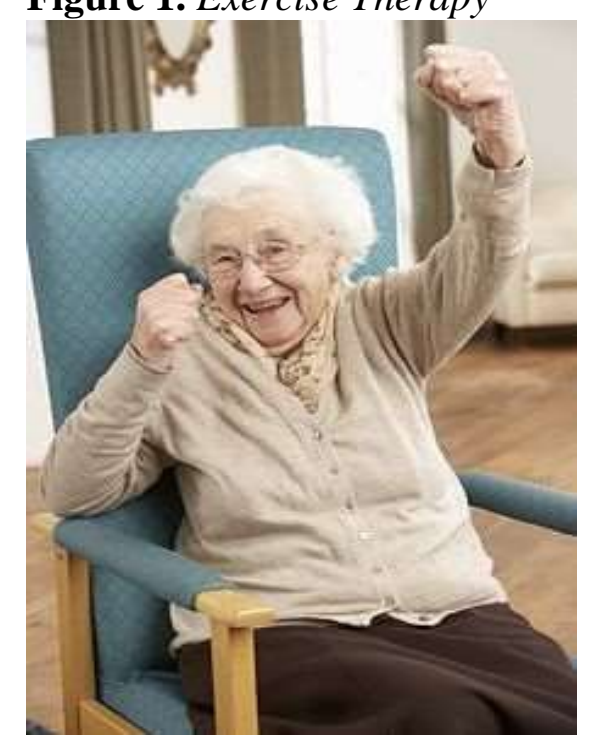


Figure 2. Group Exercise Therapy

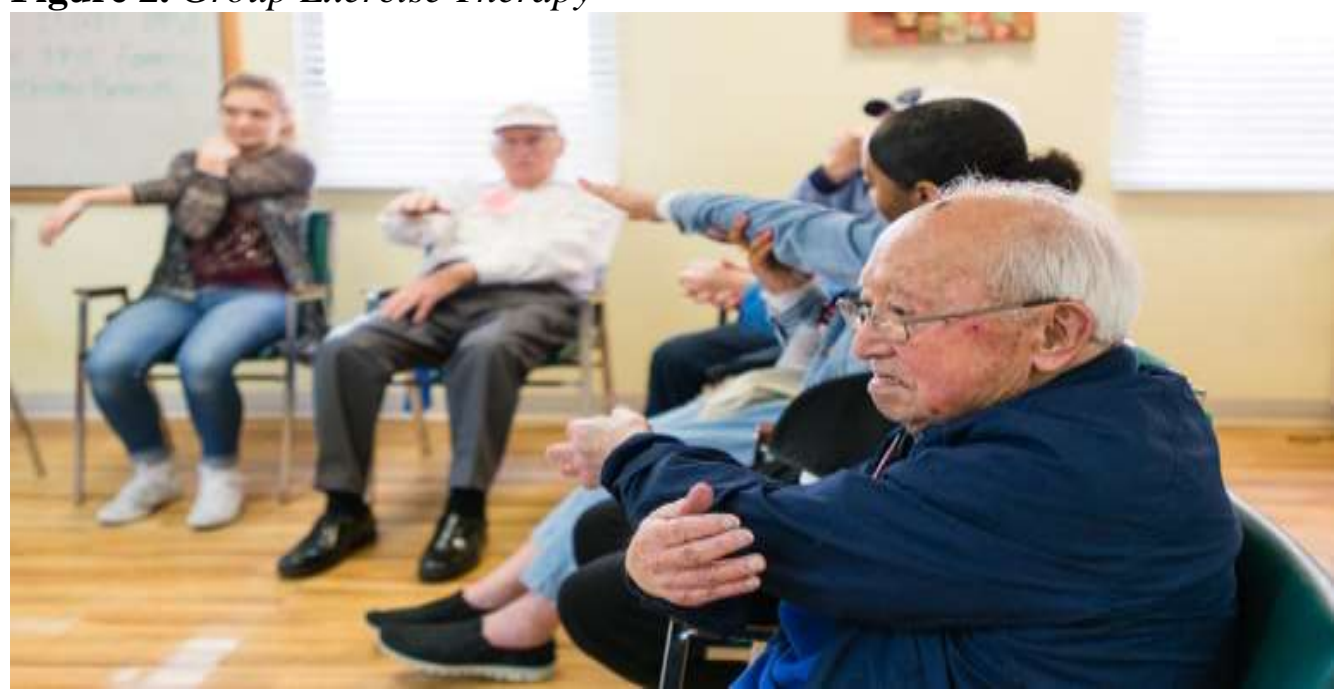

Blackburn and Bradshaw (2014) examined 6 randomized controlled trials that assessed the benefit of music therapy on patients with dementia as determined by the Mini-Mental State Exam (Figure 3). These individuals lived in residential care settings in Great Britain. Although it is suggested that music therapy may improve cognitive function and decrease anxiety, depression and agitation in older adults with dementia, the true effectiveness of music therapy remains unclear due to methodological issues of existing studies and lack of reports on which specific aspects of the intervention contribute to its effectiveness. However, music therapy is still recommended as a safe, no cost supplemental intervention to address anxiety and agitation in dementia.

\section{Figure 3. Music Therapy}

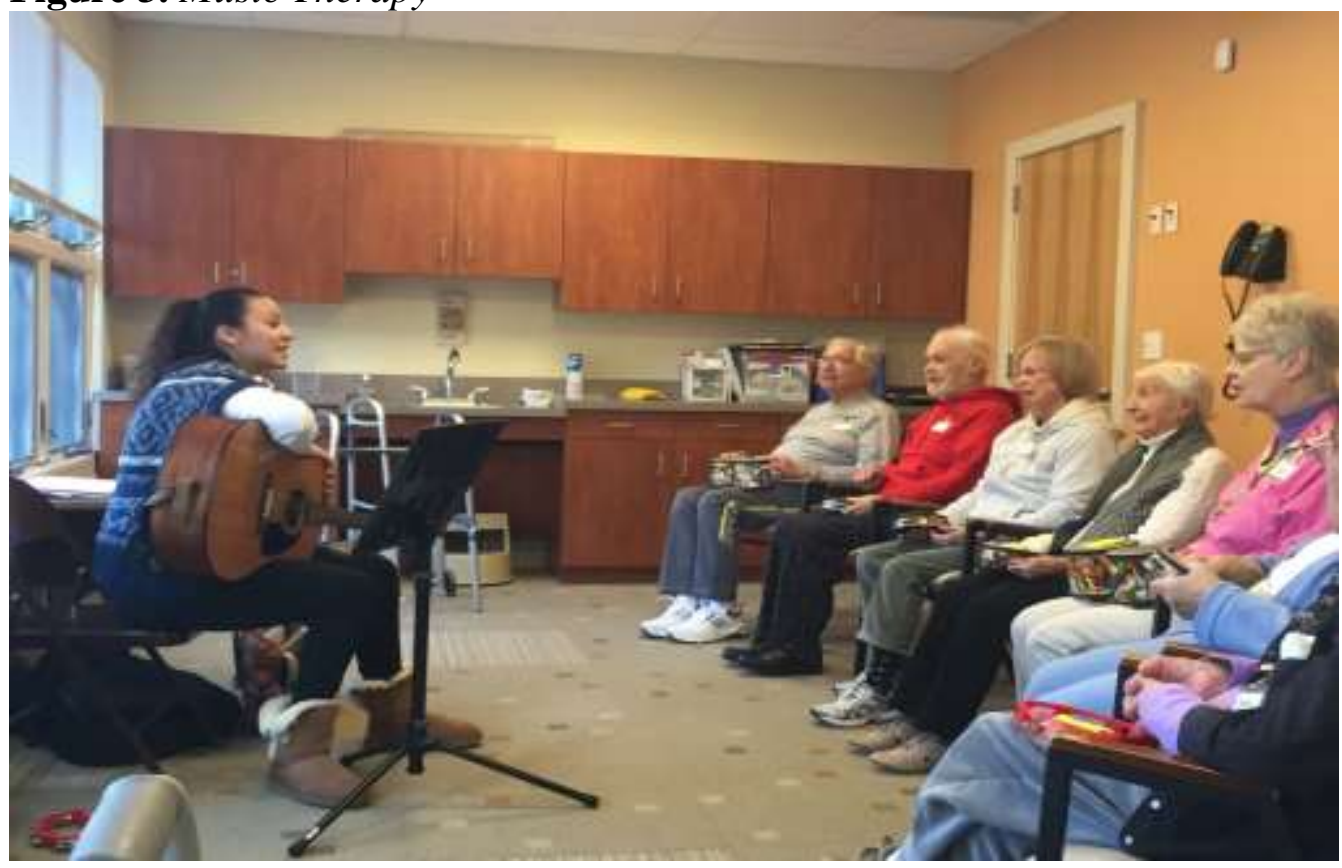


Similarly, Nair et al. (2011) studied the effectiveness of Baroque music on aggressive behaviors of residents in two nursing home units in Australia who scored on the Mini-Mental State Examination 15/30 or below. The researchers chose Baroque music based on music characteristics that mimic the human heart beat and prior reports that Baroque music enhances alpha brain waves in the brain, thus hypothesizing that this kind of music will have calming effects on agitated behaviors. Results showed that Baroque music increased agitation while the music was playing but the effect did not carry over afterwards. They concluded that although volume, individual resident music preferences and speed of music might have been factors that contributed to the results, their findings support existing documentation that music influences behavior significantly.

Table 2. Interventions to Address Challenging Behaviors in Dementia

\begin{tabular}{|c|c|c|c|}
\hline Nopharmacological & $\begin{array}{c}\text { Milieu } \\
\text { Management }\end{array}$ & Pharmacological & Other Modalities \\
\hline $\begin{array}{l}\text { Communication } \\
\text { Approaches: } \\
\text { Reminiscing, sing- } \\
\text { along, music, reality } \\
\text { orientation, short } \\
\text { sentences, simple } \\
\text { directions, maintain } \\
\text { routine and consistency, } \\
\text { reality orientation } \\
\text { Affective Approaches: } \\
\text { Positive attitude } \\
\text { Calm demeanor } \\
\text { Smile } \\
\text { Tactile Approaches: } \\
\text { Use of toolbox } \\
\text { Pet Therapy } \\
\text { Doll Therapy } \\
\text { Tactile stimulation } \\
\text { Multi-sensory } \\
\text { stimulation } \\
\text { Sensory Approaches: } \\
\text { Aromatherapy } \\
\text { Massage } \\
\text { Touch } \\
\text { Music Therapy } \\
\text { Physical Needs } \\
\text { Approaches: } \\
\text { Bathroom schedule } \\
\text { Nourishment } \\
\text { Hydration } \\
\text { Sleep Hygiene } \\
\text { Pain Assessment and } \\
\text { Intervention } \\
\text { Room Temperature } \\
\text { Regulation } \\
\text { Suitable Clothing }\end{array}$ & $\begin{array}{c}\text { Environmental } \\
\text { Safety } \\
\text { Environmental } \\
\text { Modification } \\
\text { Opportunities for } \\
\text { Physical Activity } \\
\text { Possibilities for } \\
\text { Wandering } \\
\text { Stimulation and } \\
\text { Noise Reduction } \\
\text { Opportunities for } \\
\text { Socialization } \\
\text { Neighborhoods } \\
\text { Memory Aids } \\
\text { Memory Corners } \\
\text { Work Stations }\end{array}$ & $\begin{array}{c}\text { Acetylcholinesterase } \\
\text { Inhibitors } \\
\text { (Antidementia Drugs) } \\
\text { Antidepressants } \\
\text { Antipsychotics } \\
\text { Mood Stabilizers }\end{array}$ & $\begin{array}{c}\text { Bright Light } \\
\text { Therapy } \\
\text { Electroconvulsive } \\
\text { Therapy (ECT) } \\
\text { Use of Physical } \\
\text { Restraints } \\
\text { Transcranial } \\
\text { Magnetic } \\
\text { Stimulation (TMS) }\end{array}$ \\
\hline
\end{tabular}


There is limited research examining the effectiveness of Animal-Assisted Therapy in patients suffering from dementia (Figure 4). The effectiveness of a dog-assisted intervention to address challenging behaviors in eight Swedish nursing homes involving 33 residents was studied by Nordgren and Engstrom (2014). Participants received a total of 10 sessions of dog exposure lasting up to an hour once to twice a week as opposed to a control group. It was found that there was no statistically significant improvement post intervention, however, the researchers commented on promising immediate trends showing decrease in nonaggressive behaviors. It was concluded that dog-assisted interventions may be beneficial as a complementary approach to medication to decrease behavioral symptomatology and promote social interaction. Kramer et al. (2009) introduced AIBO, a robotic dog to 18 nursing home residents with dementia and compared resident initiated interaction, smiles, laughs, and hand gesticulations to another group spending time with a person and a third group spending time with a live dog. It was concluded that although residents in all three groups were more socially interactive than before receiving the intervention, there were no statistically significant differences between the three groups in the areas of verbal and non-verbal behaviors including touch, smiles, hand gestures, and conversations, thus supporting the benefit of social interaction resulting from individualized ongoing stimulation.

\section{Figure 4. Animal Assisted Therapy}

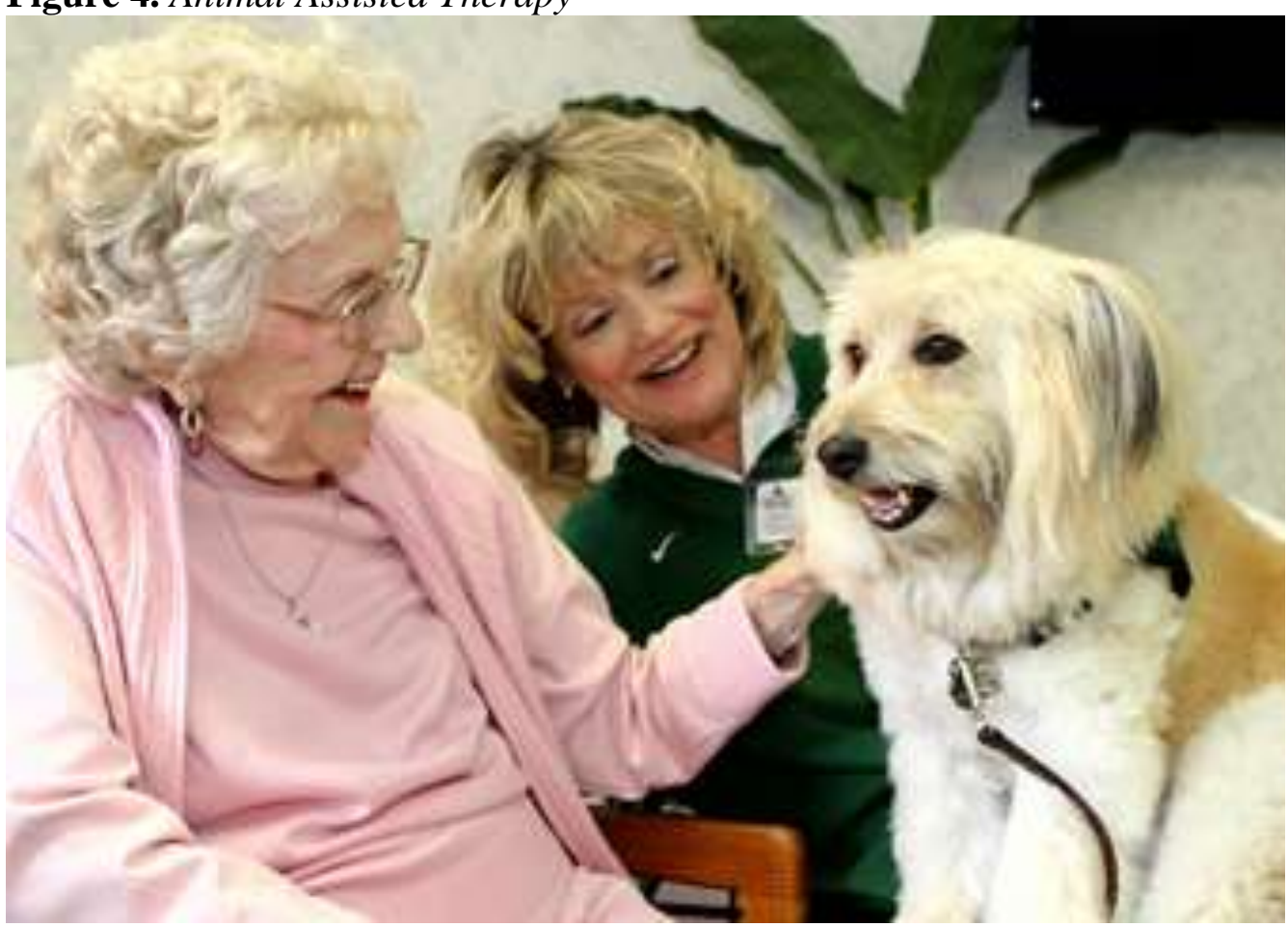


Table 3. Non-Pharmacological Intervention Studies to Address Dementia Challenging Behaviors

\begin{tabular}{|c|c|c|c|c|c|c|}
\hline Author(s) & Country/ Setting & Method & $\begin{array}{c}\text { Sample } \\
(\mathbf{N})\end{array}$ & Measures & Intervention & Findings \\
\hline Kramer et al. (2009) & $\begin{array}{l}\text { USA, } \\
\text { Nursing Home }\end{array}$ & $\begin{array}{l}\text { Behavioral } \\
\text { observational } \\
\text { approach }\end{array}$ & $\begin{array}{l}18 \text { female } \\
\text { residents }\end{array}$ & $\begin{array}{l}\text { Number of behaviors } \\
\text { per intervention (eye } \\
\text { contact, laughs, } \\
\text { smiles, hand gestures, } \\
\text { conversations, touch) }\end{array}$ & $\begin{array}{c}\text { Visit by robotic "Pet" } \\
\text { AIBO, visit by a person, } \\
\text { visit by a person and a live } \\
\text { dog }\end{array}$ & $\begin{array}{c}\text { All three types of visits } \\
\text { stimulated social interaction } \\
\text { with AIBO eliciting more and } \\
\text { longer interactions }\end{array}$ \\
\hline $\begin{array}{l}\text { Nordgren and } \\
\text { Engstrom } \\
(2014)\end{array}$ & $\begin{array}{l}\text { Sweden, Nursing } \\
\text { Homes }\end{array}$ & $\begin{array}{l}\text { Quasi-experimental, } \\
\text { pre/post-test design }\end{array}$ & $\begin{array}{l}33 \\
\text { residents }\end{array}$ & $\begin{array}{l}\text { CMAI and MDDAS } \\
\text { standardized } \\
\text { caregiver rating } \\
\text { questionnaires } \\
\end{array}$ & $\begin{array}{l}\text { Ten sessions of dog } \\
\text { assisted intervention } 45-60 \text {, } \\
\text { once or twice a week }\end{array}$ & $\begin{array}{c}\text { Aggressive behaviors decreased } \\
\text { immediately post intervention in } \\
\text { the treatment group but not } \\
\text { overtime }\end{array}$ \\
\hline $\begin{array}{l}\text { Backhouse et al. } \\
\text { (2014) }\end{array}$ & $\begin{array}{l}\text { England, } \\
\text { Nursing Homes }\end{array}$ & $\begin{array}{l}\text { Postal Survey to } \\
\text { Care Managers }\end{array}$ & $\begin{array}{l}299 \text { Care } \\
\text { Managers }\end{array}$ & $\begin{array}{l}\text { Survey developed by } \\
\text { authors }\end{array}$ & $\begin{array}{l}\text { Observed challenging } \\
\text { behaviors and approaches } \\
\text { to address them }\end{array}$ & $\begin{array}{c}\text { Reminiscence and music therapy } \\
\text { were the most utilized non- } \\
\text { pharmacological } \\
\text { approaches }\end{array}$ \\
\hline Nair et al. (2011) & $\begin{array}{c}\text { Australia } \\
\text { Nursing Home }\end{array}$ & Observational Pilot & 75 residents & Behavioral chart & $\begin{array}{c}\text { Assessed effect of Baroque } \\
\text { music on challenging } \\
\text { behaviors }\end{array}$ & $\begin{array}{l}\text { Baroque music seemed to have } \\
\text { escalated challenging behaviors }\end{array}$ \\
\hline Bauer et al. (2015) & $\begin{array}{l}\text { Australia } \\
\text { Nursing Home }\end{array}$ & $\begin{array}{c}\text { Descriptive } \\
\text { observational method }\end{array}$ & 16 residents & $\begin{array}{l}\text { Wilcoxon signed- } \\
\text { rank test }\end{array}$ & $\begin{array}{l}\text { Observed resident } \\
\text { responses from intervention } \\
\text { (Snoezelen) and control } \\
\text { group (best practice) }\end{array}$ & $\begin{array}{l}\text { No significant differences } \\
\text { between Snoezelen and "best } \\
\text { practice" interventions }\end{array}$ \\
\hline $\begin{array}{l}\text { Bremault-Phillips et } \\
\text { al. (2015) }\end{array}$ & $\begin{array}{l}\text { Canada, } \\
\text { Practitioner } \\
\text { experiences }\end{array}$ & Qualitative study & 53 participants & $\begin{array}{l}\text { Focus groups, } \\
\text { surveys }\end{array}$ & $\begin{array}{l}\text { Explored issues related to } \\
\text { disruptive behaviors, } \\
\text { develop plan to address } \\
\text { those }\end{array}$ & $\begin{array}{l}\text { There is a need and readiness to } \\
\text { develop strategies } \\
\text { to address disruptive behaviors }\end{array}$ \\
\hline $\begin{array}{l}\text { Cohen-Mansfield et } \\
\text { al. (2011a) }\end{array}$ & $\begin{array}{l}\text { USA, MDS, PhDs, } \\
\text { NPs }\end{array}$ & Web-based survey & $\begin{array}{l}180 \text { MDs, } 36 \\
\text { PhDs, } 89 \text { NPs }\end{array}$ & $\begin{array}{l}\text { Close-ended } \\
\text { questionnaire }\end{array}$ & $\begin{array}{l}\text { Assessed health care } \\
\text { provider knowledge and } \\
\text { attitude on how to address } \\
\text { challenging behaviors non- } \\
\text { Pharmacologically }\end{array}$ & $\begin{array}{c}\text { PhDs and NPs had greater } \\
\text { knowledge on how to address } \\
\text { behaviors nonpharmacologically } \\
\text { than MDs. } \\
\text { PhDs, NPs, and MDs, attitudes } \\
\text { supported the use of non- } \\
\text { pharmacological approaches, } \\
\text { with NPs scoring the highest }\end{array}$ \\
\hline
\end{tabular}




\begin{tabular}{|c|c|c|c|c|c|c|}
\hline $\begin{array}{l}\text { Cohen-Mansfield et } \\
\text { al. }(2011 \mathrm{~b})\end{array}$ & $\begin{array}{l}\text { USA, MDs, PhDs, } \\
\text { NPs }\end{array}$ & Web-based survey & $\begin{array}{l}180 \text { MDs, } 38 \\
\text { PhDs, } 100 \text { NPs }\end{array}$ & $\begin{array}{l}\text { Close ended } \\
\text { questionnaire }\end{array}$ & $\begin{array}{l}\text { Gathered information on } \\
\text { assessment and treatment } \\
\text { of behavioral problems in } \\
\text { nursing home residents }\end{array}$ & $\begin{array}{l}\text { PhDs used more frequently } \\
\text { assessment instruments, MDs } \\
\text { and PhDs utilized similar } \\
\text { behavioral and pharmacological } \\
\text { interventions }\end{array}$ \\
\hline Cooke et al. (2010) & $\begin{array}{l}\text { Australia, nursing } \\
\text { home residents }\end{array}$ & $\begin{array}{l}\text { Randomized cross- } \\
\text { over design with } \\
\text { control groups }\end{array}$ & $\begin{array}{l}47 \text { nursing home } \\
\text { residents with } \\
\text { mild to moderate } \\
\text { dementia }\end{array}$ & $\begin{array}{l}\text { Short Form Cohen- } \\
\text { Mansfield Inventory, } \\
\text { Rating Anxiety in } \\
\text { Dementia Scale }\end{array}$ & $\begin{array}{l}\text { Live, familiar song-singing } \\
\text { with musicians versus } \\
\text { reading activity }\end{array}$ & $\begin{array}{l}\text { Music did not improve anxiety } \\
\text { and agitation }\end{array}$ \\
\hline Deudon et al. (2009) & $\begin{array}{l}\text { France, nursing } \\
\text { home residents }\end{array}$ & $\begin{array}{l}\text { Randomized } \\
\text { intervention with a } \\
\text { control group }\end{array}$ & 306 residents & $\begin{array}{l}\text { Cohen-Mansfield } \\
\text { Agitation Inventory, } \\
\text { Observational Scale }\end{array}$ & $\begin{array}{c}\text { Assessed aggressive } \\
\text { behaviors post staff training } \\
\text { on handling challenging } \\
\text { behaviors }\end{array}$ & $\begin{array}{l}\text { Intervention group experienced } \\
\text { decrease in aggressive behaviors } \\
\text { post intervention with a carry- } \\
\text { over effect } 3 \text { months later }\end{array}$ \\
\hline Drapeau et al. (2009) & $\begin{array}{l}\text { Canada, } \\
\text { Outpatients with } \\
\text { dementia }\end{array}$ & Case control study & $\begin{array}{l}7 \text { patients with } \\
\text { dementia and } 16 \\
\text { healthy older } \\
\text { adults }\end{array}$ & $\begin{array}{l}\text { Standardized neuro } \\
\text { psychological testing }\end{array}$ & $\begin{array}{l}\text { Emotional reactions were } \\
\text { recorded given } 56 \text { novel } \\
\text { film clips }\end{array}$ & $\begin{array}{l}\text { Dementia individuals expressed } \\
\text { impaired recognition from the } \\
\text { face but auditory emotions were } \\
\text { present }\end{array}$ \\
\hline Fossey et al. (2006) & $\begin{array}{l}\text { Great Britain, } \\
\text { nursing home } \\
\text { residents }\end{array}$ & $\begin{array}{l}\text { Cluster randomized } \\
\text { trial }\end{array}$ & 138 residents & $\begin{array}{l}\text { Cohen-Mansfield } \\
\text { Agitation Inventory }\end{array}$ & $\begin{array}{l}\text { Trained staff over } 10 \\
\text { months to utilize non } \\
\text { Pharmaco } \\
\text { logical interventions to } \\
\text { address agitation } \\
\end{array}$ & $\begin{array}{l}\text { Staff training reduced the use of } \\
\text { medications to address agitation. } \\
\text { Results were sustained for } 12 \\
\text { months }\end{array}$ \\
\hline Sung et al. (2010) & $\begin{array}{l}\text { Taiwan, } \\
\text { Nursing home } \\
\text { residents }\end{array}$ & $\begin{array}{l}\text { Quasi-experimental } \\
\text { pre-posttest design }\end{array}$ & 52 residents & $\begin{array}{l}\text { Rating Anxiety in } \\
\text { Dementia }\end{array}$ & $\begin{array}{l}\text { Staff provided care with } \\
\text { preferred music to the } \\
\text { control group versus care } \\
\text { without music }\end{array}$ & $\begin{array}{l}\text { The intervention group } \\
\text { experienced significantly lower } \\
\text { anxiety levels }\end{array}$ \\
\hline $\begin{array}{l}\text { Khan and Curtice } \\
\text { (2011) }\end{array}$ & $\begin{array}{l}\text { Great Britain, } \\
\text { nursing home } \\
\text { residents }\end{array}$ & Qualitative study & $\begin{array}{l}63 \text { nursing home } \\
\text { residents }\end{array}$ & $\begin{array}{l}\text { Questionnaire to } \\
\text { elicit themes }\end{array}$ & $\begin{array}{l}\text { Trained care home staff to } \\
\text { restrict pharmacological } \\
\text { interventions for agitation }\end{array}$ & $\begin{array}{l}\text { A total of } 14 \text { patients were stable } \\
\text { and discharged post intervention }\end{array}$ \\
\hline $\begin{array}{l}\text { Nicholls et al. } \\
(2013)\end{array}$ & $\begin{array}{l}\text { Australia, family } \\
\text { members of } \\
\text { nursing home } \\
\text { demented } \\
\text { individuals }\end{array}$ & $\begin{array}{l}\text { Three-phase mixed } \\
\text { method design }\end{array}$ & $\begin{array}{l}31 \text { family } \\
\text { caregivers }\end{array}$ & $\begin{array}{l}\text { Semi-structured focus } \\
\text { group interviews }\end{array}$ & $\begin{array}{l}\text { Pre, post evaluation of } \\
\text { "high touch" intervention } \\
\text { based on the End-of-Life } \\
\text { Namaste Care program }\end{array}$ & $\begin{array}{l}\text { Residents and families felt more } \\
\text { relaxed and comfortable in their } \\
\text { interactions with each other }\end{array}$ \\
\hline
\end{tabular}




\begin{tabular}{|c|c|c|c|c|c|c|}
\hline $\begin{array}{l}\text { Oppikofer and } \\
\text { Geschwindner (2014) }\end{array}$ & $\begin{array}{l}\text { Switzerland, } \\
\text { nursing home } \\
\text { residents }\end{array}$ & Pre- post design & 67 residents & $\begin{array}{l}\text { Cohen-Mansfield } \\
\text { Agitation Inventory, } \\
\text { Pittsburgh Agitation } \\
\text { Scale }\end{array}$ & $\begin{array}{l}\text { Implementation of nursing } \\
\text { interventions to decrease } \\
\text { agitation }\end{array}$ & $\begin{array}{c}\text { Five nursing interventions (noise } \\
\text { reduction, ADLs, fluids, } \\
\text { communication, walking) } \\
\text { greatly decreased agitated } \\
\text { behaviors }\end{array}$ \\
\hline $\begin{array}{l}\text { Palm et al. } \\
\text { (2014) }\end{array}$ & $\begin{array}{l}\text { Germany, nursing } \\
\text { home residents }\end{array}$ & $\begin{array}{l}\text { Multi method pre, } \\
\text { post-test }\end{array}$ & 145 residents & $\begin{array}{l}\text { DemCare-Q } \\
\text { questionnaire }\end{array}$ & $\begin{array}{c}\text { Instrument development to } \\
\text { assess non-pharmacological } \\
\text { interventions } \\
\end{array}$ & $\begin{array}{l}\text { Content validity established, } \\
\text { proposed additional testing to } \\
\text { test further instrument reliability }\end{array}$ \\
\hline $\begin{array}{l}\text { Spagnolo et al. } \\
(2015)\end{array}$ & $\begin{array}{l}\text { Italy, outpatient } \\
\text { individuals with } \\
\text { dementia }\end{array}$ & $\begin{array}{c}\text { Retrospective } \\
\text { Observational study }\end{array}$ & 36 patients & $\begin{array}{l}\text { Cumulative Illness } \\
\text { Rating Scale, Mini } \\
\text { Mental Status } \\
\text { Examination, } \\
\end{array}$ & $\begin{array}{l}\text { 3R Mental Stimulation } \\
\text { intervention }\end{array}$ & $\begin{array}{l}\text { There was a decrease in } \\
\text { challenging behaviors, along } \\
\text { with caregiver distress }\end{array}$ \\
\hline Toba et al. (2014) & $\begin{array}{l}\text { Japan, newly } \\
\text { admitted nursing } \\
\text { home residents }\end{array}$ & $\begin{array}{l}\text { Non randomized } \\
\text { study design }\end{array}$ & $\begin{array}{l}158 \text { residents in } \\
\text { the intervention } \\
\text { group and } 54 \text { in } \\
\text { the control group }\end{array}$ & $\begin{array}{l}\text { Mini Mental Status } \\
\text { Examination, } \\
\text { Observational } \\
\text { Assessments, } \\
\text { Dementia } \\
\text { Disturbance Scale }\end{array}$ & $\begin{array}{l}\text { Intensive rehabilitation } \\
\text { individual activity } \\
\text { interventions }\end{array}$ & $\begin{array}{l}\text { Improvement in cognitive } \\
\text { function and behaviors }\end{array}$ \\
\hline $\begin{array}{l}\text { Van Mierlo et al. } \\
(2015)\end{array}$ & $\begin{array}{l}\text { Netherlands, newly } \\
\text { admitted nursing } \\
\text { home residents }\end{array}$ & $\begin{array}{c}\text { Qualitative } \\
\text { explorative design } \\
\text { with semi-structured } \\
\text { interviews }\end{array}$ & 22 interviews & $\begin{array}{l}\text { Mini Mental Status } \\
\text { Examination, Neuro } \\
\text { psychiatric Inventory }\end{array}$ & $\begin{array}{l}\text { Family and staff education } \\
\text { education on how to deal } \\
\text { with difficult behaviors of } \\
\text { individuals transitioning } \\
\text { from the community to the } \\
\text { nursing home family } \\
\text { support }\end{array}$ & $\begin{array}{l}\text { Intervention increased } \\
\text { awareness for both staff and } \\
\text { families of challenging } \\
\text { behaviors }\end{array}$ \\
\hline Tournier et al. (2017) & $\begin{array}{l}\text { France, nursing } \\
\text { home residents }\end{array}$ & $\begin{array}{l}\text { Pre-, post- } \\
\text { intervention }\end{array}$ & 15 residents & $\begin{array}{l}\text { Mini Mental Status } \\
\text { Examination, } \\
\text { systematic } \\
\text { assessment of } \\
\text { observed behaviors } \\
\end{array}$ & Trainer-dog pair sessions & $\begin{array}{c}\text { Modest effect on resident } \\
\text { behavioral scores, but significant } \\
\text { improvement in caregiver stress } \\
\text { scores }\end{array}$ \\
\hline
\end{tabular}




\section{Pharmacological Interventions}

Pharmacological agents are considered the last choice of treatment to address unsafe, challenging behaviors in dementia, that include pacing, restlessness, irritability, extreme anxiety, agitation and aggression. Although approximately 40$60 \%$ of individuals who experience the above behaviors are nursing home residents, it has been reported that an additional $20 \%$ experience those behaviors in the community (Zeller et al. 2016). The safety and efficacy of available medications is considered controversial and clinicians are urged to exercise caution given the severity of possible adverse reactions of most medications. Moreover, dose variations, diversity in assessment scales and limited data reporting from failed trials in addition to population heterogeneity when it comes to drug responses create further issues and limit our knowledge when it comes to medication management of behaviors and symptomatology (Anand et al. 2014).

\section{$\underline{\text { Acetylcholinesterase Inhibitors and Antidepressants }}$}

Although the benefit of acetylcholinesterase inhibitors has been consistently discussed in the literature of the past two decades, the efficacy of medications such as memantine and donepezil to address aggression agitation, and irritability has not been consistent. Furthermore, methodological limitations, small sample sizes, lack of assessment tools, and variance in the use of control groups complicate the picture even further (Cankurtaran 2014). Conservative benefits of antidepressants, with SSRIs being the most preferable option, have been associated with improvement in agitation, and are better tolerated than typical and atypical antipsychotics. Research studies still need to explore safety issues associated with long-term use (Cankurtaran 2014, Ballard and Corbett 2010).

\section{$\underline{\text { Antipsychotics }}$}

The use of typical and atypical antipsychotic medications has been discussed primarily over the past two decades in managing aggressive behaviors. Small placebo-controlled trials have focused on the effectiveness of controlling agitation and aggression, yet the use of antipsychotics and concerns over the possibility of cerebrovascular complications, prolonged QT intervals, dysthonias, Parkinsonian presentation and concerns about increase in mortality rates have limited intervention periods and make the use of antipsychotics controversial amongst prescribers (Ballard and Corbett 2010). In a prospective clinical trial Mintzer et al. (2006) found risperidone to be significantly effective in addressing psychotic presentation, whereas the efficacy of olanzapine was not supported by all reviewed studies (Schneider et al. 2006, Mintzer et al. 2006, Sultzer et al. 2008). Additionally, quetiapine did not address agitation adequately (Ballard et al. 2005), yet, aripiprazole provided significant amelioration in aggression in another clinical trial (Streim et al. 2008). 
$\underline{\text { Mood Stabilizers and Antiepileptics }}$

Preliminary results from small studies offer conservative benefits for the use of antiepileptic agents to decrease behavioral disturbances, with some agents showing little to no effect in addressing those behaviors (Hungerford et al. 2014). A small number of studies have modestly supported carbamazepine, gabapentin, topiramate and lamotrigine in managing agitation and aggression in some patients, whereas valproate and lithium provided very limited or no effectiveness in managing behaviors (Yeh and Ouyang 2012, Amann et al. 2009, Ng et al. 2009, Desai and Grossberg 2001). Small sample sizes, inconsistencies in the use of measurement scales, use of subjective measurements, low reliability of outcomes, medication adverse reactions and drug to drug interactions in the elderly populations necessitate for close attention and conservative approaches when it comes to the use of these medications to manage aggressive behaviors.

\section{Other Treatment Modalities}

\section{Electroconvulsive Therapy (ECT)}

Although ECT has been widely utilized with great effectiveness in the treatment of mood depressive disorders in the general population, little has been reported on the effectiveness of this treatment modality to control agitation and aggression in dementia. Yet, ECT is considered to be an attractive option, especially when antipsychotics produce limited results in the area of symptom management, or are associated with serious side effects. Despite its safety and reported effectiveness ECT is considered an off-label, last resort approach in absence of treatment effectiveness of other interventions (Ujkaj 2015). Research findings have been consistently promising over the past decades. For instance, Bang et al. (2008), Wu et al. (2010), Kerner and Prudic (2014), Holmberg et al. (1996) reported improvement in depression, and agitation in patients suffering from dementia.

Consistent with those findings in a more recent study, encouraging results from the use of ECT were reported by Acharya et al. (2015) who followed a group of 23 individuals receiving ECT as the primary intervention to address agitation and aggression. It was found that a total of $87 \%$ of the participants tolerated the procedure well and experienced statistically significant reduction in aggression and agitation from baseline. The remaining participants had to discontinue the study primarily because of issues unrelated to ECT such as development of infection (9\%), new cardiac diagnosis (4\%), death (4\%). Only two participants $(9 \%)$ did not benefit from treatment. Similarly to other ECT studies, this study was also limited due to the small sample size, the heterogeneity of dementia diagnoses and the lack of a control group. 


\section{$\underline{\text { Transcranial Magnetic Stimulation (TMS) }}$}

The effectiveness of TMS has been studied in depression with promising effectiveness. Existing studies in dementia have found that TMS may improve cognitive function and verbal communication (Wu et al. 2015, George et al. 2010, Hoogendam et al. 2010, Cotelli et al. 2008, Cotelli et al. 2011). In a randomized trial by Wu et al. (2015) the effectiveness of high frequency transcranial magnetic stimulation (rTMS) in conjunction with standard antipsychotic medications was assessed in a group of 27 participants with a Mini-Mental State Examination (MMSE) of less than 24 and compared to a control group receiving only antipsychotic medication over a period of 4 weeks. It was found that the intervention group experienced statistically significant improvement in the areas of anxiety, phobias, sleep, cognitive function, mood instability, restlessness, aggression and agitation. Although the results on the effectiveness of rTMS and antipsychotic medication were promising, the researchers acknowledged limitations related to the small sample size, and lack of data discussing long-term effectiveness, as well as lack of direction for the use of booster sessions.

\section{Bright Light Therapy (BLT)}

The use of BLT has been studied with a number of conditions. The effectiveness of this intervention is rather inconclusive when addressing agitation in older adults with dementia (Hanford and Figueiro, 2013). The effects of daily administration of BLT for one hour over a period of 11 weeks were studied in a randomized trial by Dowling et al. (2005). One group received the intervention in the morning, another one in the afternoon and the control group received regular indoor light. The researchers reported statistically significant differences between the three groups in the areas of aggression and agitation, thus supporting the effectiveness of BLT. In another randomized controlled trial participants in the intervention group were exposed to 10,000 lux intensity light, whereas controls were exposed to standard 100 lux fluorescent light, for a period of two weeks, for two hours a day. It was found that participants in the intervention group experienced improved sleep and decreased agitation, however, the findings were not statistically significant (Dowling et al. 2005).

\section{Nursing Implications}

The literature overwhelmingly supports a combination of treatment approaches that individuals suffering from the illness can benefit from. Nurses have an ongoing involvement in the care of these individuals not only in long term care facilities and the community, but also in the hospital setting. Providing personcenter care interventions greatly increases the chances to successfully address challenging behaviors that have the potential to escalate to aggressive presentation (Stein-Parbury et al. 2012). The enhancement of cognitive function through activities such as socialization, reminiscence, support of activities of daily living, 
are just a few basic examples that promote environmental structure, reorientation and a sense of security, which in return may contribute to the decrease of challenging behaviors (Cohen-Mansfield et al. 2011b).

Although major advances in the area of neuropsychiatry have enabled health care providers to understand the biological basis of challenging behaviors and aggression associated with Alzheimer's disease and related dementias, there is still much to be discovered. Yet, nurses are in a unique position to know their patients' personality characteristics and abilities, thus being able to implement communication strategies, activities, and other related approaches to promote a culture of safety and self-worth, where everyone feels protected. By modifying the milieu, eliminating loud, frightening noises, clutter, or overstimulation, but also making clinical judgement calls when the assistance of medication may be helpful, the intensity of a challenging behavior can be significantly decreased (Keltner et al. 2011). This in return can promote quality of life for the affected individual overtime, but also decrease health care provider and family caregiver burnout with the ultimum goal of delaying and in some cases even preventing nursing home placement.

\section{Conclusions and Future Recommendations}

Our understanding of the biological basis of disturbing behaviors in dementia with the most difficult ones of agitation and aggression is limited and the pathophysiology of the illness is continually changing. The exact process of the disease process is still unclear. New findings often times replace previous knowledge about the process. For instance, neuron damage now is believed to be a rather continuous and ongoing process caused from tangles (Anand et al. 2014). A complex casqued of mechanisms affecting mitochondrial dysfunction, and contributing to chronic oxidative stress, inflammation, hormonal imbalances, neurofibrillary tangle accumulation, in addition to genetic abnormalities, seem to have a significant contribution in the disease process (Hungerford et al. 2014). Currently, the focus of pharmacological and non-pharmacological treatments is on addressing specific symptomatology.

The evidence of successful pharmacological management of disruptive behaviors in dementia is inconclusive and study outcomes are limited by a variety of uncontrolled variables and sampling issues. Cholinesterase inhibitors, antidepressants, typical and atypical antipsychotics, benzodiazepines, mood stabilizers and other pharmacological interventions have shown various degrees of effectiveness. Upcoming research presents some innovative approaches, which although at the infancy stage, appear to be promising. Amongst those, immunetherapy, anti-inflammatory therapy, antioxidant supplementation, DNA vaccination are attractive options to consider. In addition, great emphasis is placed on understanding the disease process correctly, as well as the drug's bioavailability and pharmacokinetics (Anand et al. 2014).

Yet, health care providers have been more successful when including psychosocial and behavioral interventions than medication alone. In hospital and 
nursing home settings, nursing staff can utilize a variety of behavioral interventions to redirect inappropriate or challenging behaviors, provide structure and purpose and promote self-esteem. Individual and group activities that provide mental stimulation draw from familiar past roles and support self-worth can diminish disruptive behaviors even for a brief period of time. Activities such as music therapy, sing along, reminiscing, aromatherapy, movie night, grooming group, pet therapy, exercise, are just a few options that are popular treatment interventions and are considered safe and well tolerated. Future research can explore the effectiveness of specific behavioral interventions and medications that are successful in addressing specific disruptive behaviors taking into consideration individuals' cultural background. In addition, involving family members in such activities, may allow for continuation of successful interventions at home for community dwelling patients with the ultimate benefits of improved quality of life and perhaps delay in nursing home placement. Moreover, new problem solving health care provided approaches such as the "DICE" approach (Describe, Investigate, Create, Evaluate) can assist in implementing a plan of care that focuses on individual patient needs, thus allowing for more successful management of behaviors (Kales et al. 2014b). Finally, research studies on caregiver direct involvement in activities for the affected person can provide new insights into the areas of caregiver physiological and psychological health.

\section{References}

Acharya D, Harper HG, Achtyes ED et al. (2015) Safety and Utility of Acute Electroconvulsive Therapy for Agitation and Aggression in Dementia. International Journal of Geriatric Psychiatry 30(3): 265-273. DOI=10.1002/gps.4137.

Amann B, Pantel J, Grunze H, Vieta E, Colom F, Gonzalez-Pinto A et al. (2009) Anticonvulsants in the Treatment of Aggression in the Demented Elderly: An Update. Clinical Practice in Epidemiology and Mental Health 5: 14.

Anand R, Gill KD, Mahdi AA (2014) Therapeutics of Alzheimer's Disease: Past, Present and Future. Neuropharmacology 76: 27-50. DOI= dx.doi.org/10.1016/j.neuropharm. 2013.07.004.

Backhouse T, Killett A, Penhale B, Burns D, Gray R (2014) Behavioural and Psychological Symptoms of Dementia and their Management in Care Homes within the East of England: A Postal Survey. Aging Mental Health 18(2): 187-193. DOI= 10.1080/13607863.2013.819834.

Ballard C, Corbett A (2010) Management of Neuropsychiatric Symptoms in People with Dementia. CNS Drugs 24(9):729-739.

Ballard C, Margallo-Lana M, Juszczak E, Douglas S, Swann A, Thomas A, O'Brien J, Everratt A et al. (2005) Quetiapine and Rivastigmine and Cognitive Decline in Alzheimer's Disease: Randomized Double Blind Placebo Controlled Trial. British Medical Journal 16(February 2005): 874. DOI=10.1136/bmj.38369.459988.8F.

Bang J, Price D, Prentice G, Campbell J. (2008) ECT Treatment for Two Cases of Dementia-related Pathological Yelling. Journal of Neuropsychiatry and Clinical Neuroscience 20(3): 379-380.

Bauer M, Rayner JA, Tang J, Koch S, While Ch, O'Keefe F (2015) An Evaluation of Snoezelen Compared to "Common Best Practice" for allaying the Symptoms of 
Wandering and Restlessness among Residents with Dementia in aged Care Facilities. Geriatric Nursing 36(6): 462-466. DOI= http://dx.doi.org./10.1016/j.gerinurse.2015. 07.005 .

Benbow B (2014) Design Features for Resident Engagement and Meaningful Activity. Canadian Nursing Home 25(4): 4-8.

Blackburn R, Bradshaw T (2014) Music Therapy for Service users with Dementia: A Critical Review of the Literature. Journal of Psychiatric and Mental Health Nursing 21(10): 879-888.

Bremault-Phillips S, Germani T, Sacrey LA, Friesen S (2015) Managing Disruptive Behaviours Exhibited by Older Adults with Mental Health, Addictions and Neurocognitive Conditions in Alberta: A Mixed Methods Approach. Geriatric Mental Health Care 3(2): 21-27.

Cankurtaran ES (2014) Management of Behavioral and Psychological Symptoms of Dementia. Archives of Neuropsychiatry 51(4): 303-312. DOI= 10.5152/npa.2014. 7405 .

Child A, Clarke A, Fox CH, Maidment J (2012) A Pharmacy Led Program to Review Anti-Psychotic Prescribing for People with Dementia. BioMed Central Psychiatry 12(1): 155 . DOI= 1186/1471-244x-12-155.

Cohen-Mansfield J, Jensen B, Resnick B, Norris M (2011a) Assessment and Treatment of Behavior Problems in Dementia in Nursing Home Residents: A Comparison of the Approaches of Physicians, Psychologists, and Nurse Practitioners. International Journal of Geriatric Psychiatry 27(2): 135-145. DOI=10.1002/gps.2699.

Cohen-Mansfield J, Jensen B, Resnick B, Norris M (2011b) Knowledge of and Attitudes Toward Nonpharmacological Interventions for Treatment of Behavior Symptoms Associated with Dementia: A comparison of Physicians, Psychologists, and Nurse Practitioners. The Gerontologist 52(1): 34-45. DOI= 10.1093/geront/gnr081.

Cooke ML, Moyle W, Shum DH et al. (2010) A randomized Controlled Trial Exploring the Effect of Music on Agitated Behaviours and Anxiety in Older People with Dementia. Aging and Mental Health 14(8): 905-916.

Cotelli M, Manenti R, Cappa F, Zanetti O, Miniussi C (2008) Transcranial Magnetic Stimulation Improves Naming in Alzheimer Disease Patients at Different Stages of Cognitive Decline. European Journal of Neurology 15(12): 1286-1292. DOI= http:// dx.doi.org/10.1111/j.1468-1331.2008.02202.x.

Cotelli M, Calabria M, Manenti R, Rosini S, Zanetti O, Cappa SF (2011) Improved Language Performance in Alzheimer Disease following Brain Stimulation. Journal of Neurology 82(7): 794-797. DOI= http://dx.doi.org/10.1136/jnnp.2009.197848.

Derlinde RM, Stephan B, Dening T, Brayne C (2014) Instruments to Measure Behavioural and Psychological Symptoms of Dementia. International Journal of Methods in Psychiatric Research 23(1): 69-98.

Desai AK, Grossberg GT (2001) Recognition and Management of Behavioral Disturbances in Dementia. Journal of Clinical Psychiatry 3(3): 93-109.

Deudon A, Maubourguet N, Gervais X et al. (2009) Non-pharmacological Management of Behavioural Symptoms in Nursing Homes. International Journal of Geriatric Psychiatry 24(12): 1386-1395. DOI= 10.1002/gps.2275.

Dowling GA, Mastick J, Hubbard EM, Luxenberg JS, Burr RL (2005) Effect of timed Bright Light Treatment for Rest-activity Disruption in Institutionalized Patients with Alzheimer's Disease. International Journal of Geriatric Psychiatry 20(8): 738-743. DOI=10.1002/gps.1352.

Drapeau J, Gosselin N, Gagnon L et al. (2009) Emotional Recognition from Face, Voice, and Music in Dementia of the Alzheimer Type. The Neurosciences and Music III- 
Disorders and Plasticity. International Journal of Geriatric Psychiatry 20(8): 738 743. DOI=10.1111/j.1749-6632.2009.04768.x.

Duane FM, Goeman DP, Beanland J, Koch SH (2015) The Role of a Clinical Nurse Consultant Specialist. A Qualitative Evaluation. Dementia 14(4): 436-449. DOI= http://10.1177/14713012I3498759.

Fargo K, Bleiler L (2014) Alzheimer's Association Report. 2014 Alzheimer's disease Facts and Figures. Alzheimer's and Dementia 10(2): e47-e92. DOI= http://dx.doi.org/ 10.1016/j.jalz.2014.02.001.

Forrester LT, Maayan N, Orrell M, Spector AE, Buchan LD, Soares-Weiser K (2014) Aromatherapy for Dementia. Cochrane Database of Systemic Reviews. DOI=10.10 02/14651858.CD003150.pub2.

Fossey J, Ballard C, Juszczak E et al. (2006) Effects of Enhanced Psychosocial Care on Antipsychotic use in Nursing Home Residents with Severe Dementia: Cluster Randomized Trial. British Medical Journal March 2006(XXX) DOI: 10.1136/bmj. 38782.5758 68.7C.

George MS, Lisasnby SH, Avery D, McDonald WM et al. (2010) Daily Left Prefrontal Transcranial Magnetic Stimulation Therapy for Major Depressive Disorder: A Shamcontrolled Randomized Trial. Archieves of General Psychiatry 67(5): 507-516. DOI= http://dx.doi.org/10.1001/arcgenpsychiatry.2010.46.

Hanford N, Figueiro M (2013) Light Therapy and Alzheimer's Disease and Related Dementia: Past, Present, and Future. Journal of Alzheimer's Disease 33(4): 913-922.

Holmberg SK, Tariot PN, Challapalli R (1996) American Journal of Geriatric Psychiatry 4(4): 330-334. DOI= 10.1097/00019442-199622440-00007.

Hoogendam JM, Ramakers GM, DiLazzaro V (2010) Physiology of Repetitive Transcranial Magnetic Stimulation of the Human Brain. Brain Stimulation 3(2): 95118. DOI= http://dx.doi.org/10.1016/j.brs.2009.10.005.

Hungerford C, Jones T, Cleary M (2014) Pharmacological versus Non-pharmacological Approaches to Managing Challenging Behaviours for People with Dementia. British Journal of Community Nursing 19(2): 72-77.

Kales HC, Gitlin LN, Lyketsos CG (2014a) The Time is now to Address Behavioral Symptoms of Dementia. Journal of American Society on Aging 38(3): 86-95. DOI= 10.1111/jgs. 12730.

Kales HC, Gitlin LN, Lyketsos CG (2014b) Management of Neuropsychiatric Symptoms of Dementia in Clinical Settings: Recommendations from a Multidisciplinary Expert Panel. Journal of the American Geriatrics Society 62(4): 762-769.

Khan F, Curtice M (2011) Non-Pharmacological Management of Behavioural Symptoms of Dementia. British Journal of Community Nursing 16(9): 441-449. DOI=10.12968 /bjen.2011.16.9.441.

Keltner LN, Bostrom CE, McGuinness TM (2011) Psychiatric Nursing. $6^{\text {th }}$ edition. Elsevier.

Kerner N, Prudic, J (2014) Current Electroconvulsive Therapy Practice and Research in the Geriatric Population. Neuropsychiatry 4(1): 33-54.

Kramer SC, Friedman E, Bernstein PL (2009) Comparison of the Effect of Human Interaction, Animal-Assisted Therapy, and AIBO-Assisted Therapy on Long-Term Care Residents with Dementia. Anthrozoos 22(1): 43-57. DOI= 10.2752/17530370 $8 X 390464$.

Mintzer J, Greenspan A, Caers I et al. (2006) Risperidone in the Treatment of Psychosis of Alzheimer Disease: Results from a Perspective Clinical Trial. American Journal of Geriatric Psychiatry 14(3): 280-291.

Mitchell G. (2014) Use of Doll Therapy for People with Dementia: An Overview. Nursing Older People 26(4): 24-26. 
Nair B, Heim C, Krishnan C, D’Este C, Marley J, Attia J. (2011) The Effect of Baroque Music on Behavioural Disturbances in Patients with Dementia. Australian Journal on Ageing 30(1): 11-15. DOI=10.1111/j.1741-6612.2010.00439.x.

$\mathrm{Ng} \mathrm{B}$, Camacho A, Bardwell W, Sewell DD (2009) Lamotrigine for Agitation in Older Patients with DEmentia. International Psychogeriatrics 21(1): 207-208.

Nicholls D, Chang E, Johnson A, Edenborough M (2013) Touch, the Essence of Caring for People with End-stage Dementia: A Mental Health Perspective in Namaste Care. Aging and Mental Health 17(5): 571-578. DOI=http://dx,doi.org/10.1080/13607863. 2012.751581.

Nordren L and Engstrom G (2014) Effects of Dog-assisted Intervention on Behavioural and Psychological Symptoms of Dementia Nursing Older People 26(3): 31-38.

Oppikofer S, Geschwindner J (2014) Nursing Interventions in Cases of Agitation and Dementia. Dementia 13(3): 306-317. DOI=10.1177/147/30/2/2461/110.

Palm R, Kohler K, Bartholomeyczik, Holle (2014) Assessing the Application of NonPharmacological Interventions for People with Dementia in German Nursing Homes: Feasibility and Content Validity of the Dementia Care Questionnaire (DemCare-Q). BioMed Central Research Notes 7(950) DOI= 10.1186/1756-0500-7-950.

Prince M, Bryce R, Albanese E, Wimo A, Ribeiro W, Ferri CP (2013) The Global Prevalence of Dementia: A Systematic Review and Metaanalysis. Alzheimer's and Dementia 9(1): 63-75. DOI=http://dx.doi.org/10.1016/j.jalz.2012.11.007.

Rabins PV, Lyketsos CG (2011) A Commentary on the Proposed DSM Revision Regarding the Classification of Cognitive Disorders. The American Journal of Geriatric Psychiatry 19(3): 201-204. DOI= http://dx.doi.org/10.1097/JGP.0b013e $3182051 \mathrm{ac} 7$.

Schneider LS, Dagerman K, Insel, PS (2006) Efficacy and Adverse Effects of Atypical Antipsychotics for Dementia: Meta-analysis of Randomized, Placebo-controlled Trials. American Journal of Geriatric Psychiatry 14(3): 191-210.

Soares-Weiser LD (2014) Aromatherapy for Dementia. Cochrane Database of Systematic Reviews 2. DOI= 10.1002/14651858.CD003150.pub2.

Spagnolo P, Arico M, Bergamelli C et al. (2015) A Pilot Study on Behavioral and Cognitive Effects of Cognitive Stimulation Training based on 3R Mental Stimulation. The Treviso Dementia (TREDEM) Study. Neurorehabilitation 36(1): 151-156. DOI=10.3233/NRE-141202.

Stein-Parbury J, Chenoweth L, Jeon, YH, Hass M (2012) Implementating PersonCentered Care in Residential Dementia Care. Clinical Gerontologist 35(5): 404-424. DOI $=10.1080 / 07317115.2012 .702654$.

Streim JE, Porsteinsson AP, Breder CD et al. (2008) A Randomized Double-blind, Placebo-controlled Study of Aripiprazole for the Treatment of Psychosis in Nursing Home Patients with Alzheimer Disease. American Journal of Geriatric Psychiatry 16(7): 537-550.

Sultzer DL, Davis SM, Tariot PN et al. (2008) Clinical Symptom Responses to Atypical Antipsychotic Medications in Alzheimer's Disease: Phase 1 Outcomes from the CATIE-AD Effectiveness. American Journal of Psychiatry 165(7): 844-854.

Sung H-Ch, Chang AM, Lee W-L (2010) A Preferred Music Listening Intervention to Reduce Anxiety in Older Adults with Dementia in Nursing Homes. Journal of Clinical Nursing 19(7-8): 1056-1064. DOI=10.1111/j.1365-2702.2009.03016.x

Toba K, Nakamura Y, Endo $\mathrm{H}$ et al. (2014) Intensive Rehabilitation for Dementia Improved Cognitive Function and Reduced Behavioral Disturbance in Geriatric Health Service Facilities in Japan. Geriatrics and Gerontology International 14(1): 206-211. DOI=10. 1111/ggi.12080. 
Tournier I, Vives MF, Postal V (2017) Animal-Assisted Intervention in Dementia. Effects of Neuropsychiatric Symptoms on Caregivers' Distress Perceptions. Swiss Journal of Psychology 76(2): 51-58.

Ujkaj M (2015) Dementia, Agitation, and Aggression: The Role of Electroconvulsive Therapy. Psychiatric Times 25 March 2015. http://www.psychiatrictimes.com/ special-reports/dementia-agitation-and-aggression-role-electroconvulsive-therapy.

Van Den Wijngaart MA, Vernooij-Dassen MJ, Felling AJ (2007) The Influence of Stressors, Appraisal and Personal Condition on the Burden of Spousal Caregivers of Persons with Dementia. Aging Mental Health 11(6): 626-636. DOI: 10.1080/1360 7860701368463.

Van DerLinde RM, Stephan BC, Dening T, Brayne C. (2014) Instruments to Measure Behavioural and Psychological Symptoms of Dementia. International Journal of Methods in Psychiatric Research 23(1): 69-98. DOI= 10.1002/mpr.1414.

Van Mierlo LD, Van der Wiel, Meiland FJ, Van Hout HPJ, Stek ML, Droes RM (2015) Tailored Mental Health Care after Nursing Home Admission: Improving Transfers of People with Dementia with Behavioral Problems. An Explorative Study. Aging and Mental Health 19(10): 902-911. DOI= http://dx.doi.org/10.1080/13607863.2014. 977774.

Wu Q, Prentice G, Cambell JJ (2010) ECT Treatment for Two Cases of Dementia-related Aggressive Behavior. Journal of Neuropsychiatry and Clinical Neuroscience 22: E10-E11.

Wu Y, Xu W, Liu X, Xu Q, Tang L, Wu S (2015) Adjunctive Treatment with High Frequency Repetitive Transcranial Magnetic Stimulation for the Behavioral and Psychological Symptoms of Patients with Alzheimer's Disease: A Randomized, Double-blind, Sham-controlled Study. Shanghai Archives of Psychiatry 27(5): 280288. DOI= http://dx.doi.org/10.11919/j.issn.1002-0829.215107.

Yeh YC, Ouyang WC (2012) Mood Stabilizers for the Treatment of Behavioral and Psychological Symptoms of Dementia: An Update Review. Kaohsiung Journal of Medical Sciences 28(4): 185-193. DOI=10.1016/j.kjms.2011.10.025.

Zeller A, Hahn S, Needham I, Kok G, Dassen T, Halfens RJG (2016) Aggressive Behavior of Nursing Home Residents Toward Caregivers: A Systematic Literature Review. Geriatric Nursing 30(3): 174-187.

Zwijsen SA, Smalbrugge M, Eefsting JA, Gerritsen DL, Hertogh CM, Pot AM (2014) Grip on Challenging Behaviors: Process evaluation of the Implementation of a Care Program. Trials 25(15): 302. DOI= 10.1186/1745-6215-15-302. 